\title{
Nanofluidics: Viscous Dissipation in Layered Liquid Films
}

\author{
Thomas Becker and Frieder Mugele* \\ Universität Ulm, Abteilung Angewandte Physik, Albert Einstein Allee 11, D-89081 Ulm Germany
}

(Received 4 March 2003; published 17 October 2003)

\begin{abstract}
We studied the layer-by-layer collapse of molecularly thin films of a model lubricant confined between two atomically smooth substrates. The dynamics of the consecutive expulsion of four molecular layers were found to slow down with decreasing film thickness but showed no evidence for confinement-induced solidification. Using a hydrodynamic model, we show that the sliding friction of liquid layers on top of the solid substrates is approximately 18 times higher than the mutual friction between adjacent liquid layers. The latter was independent of film thickness and in close agreement with the bulk viscosity.
\end{abstract}

DOI: 10.1103/PhysRevLett.91.166104

The ongoing miniaturization of both microelectromechanical (MEMS) and microfluidic devices creates an increasing interest in transport properties of liquids near solid-liquid interfaces. For instance, the thickness of liquid lubricant layers that protect MEMS from excessive friction and wear approaches molecular dimensions [1]. In this regime, the structural and dynamical properties of liquids deviate strongly from the extrapolated bulk behavior in various respects: It was shown that the classical hydrodynamic no-slip boundary condition can be violated on the molecular scale [2]. For simple liquids confined between atomically smooth solid substrates, it was shown that the molecules arrange into layers parallel to the surfaces [3-8]. These structural modifications also affect the dynamics: At a thickness of a few molecular diameters, drainage of liquid films does not occur continuously, but in a series of discrete steps [5], usually called layering transitions. Moreover, frictional forces measured by shearing the confining walls laterally were reported to increase strongly with decreasing film thickness [6-8]. However, a consistent picture of the dynamics in fluids at the boundary between molecular motion and continuum flow has yet to emerge.

In this Letter, we analyze the dynamics of a series of consecutive layering transitions in a surface forces apparatus (SFA). We present experimental results recorded with the recently developed imaging technique for the SFA [9]. The data are interpreted in terms of a model for fluid flow in thin films, which takes into account the discrete layer structure.

The experimental setup consisted of two atomically smooth mica sheets mounted in crossed cylinder geometry (see inset of Fig. 1). The mica sheets were silvered on their back sides and thus formed a Fabry-Pérot interferometer. The instrument was illuminated with monochromatic light, the wavelength of which was adjusted to the wing of one of the transmission peaks [10]. Using a video microscope, we recorded two-dimensional video images in transmission. Inside the contact zone, where the substrates were flattened elastically, the liquid film was trapped in a pore space of constant width. In order to
PACS numbers: 68.35.Af, 68.08.-p, 68.15.+e

assure that the mica sheets were indeed atomically smooth (in particular free of contaminating nanoparticles [11]), we recleaved the surfaces immediately prior to the experiments [12] and verified the cleanliness by atomic force microscopy. For the present study, we used octamethylcyclotetrasiloxane (OMCTS), as a model liquid with nonpolar quasispherical molecules (diameter $\approx 0.9 \mathrm{~nm}$ ). OMCTS (98\% pure) was obtained from ABCR, Karlsruhe (Germany) and was used as received. The residual water content was $3 \mathrm{ppm}$, as determined by titration. After closing the SFA chamber in dry air, a droplet $(\approx 10 \mu \mathrm{L})$ was injected into the gap between the mica surfaces with a syringe. A small container of $\mathrm{P}_{2} \mathrm{O}_{5}$ inside the SFA chamber kept the atmosphere dry during the experiments. Experiments were performed at a room temperature of $(22 \pm 1)^{\circ} \mathrm{C}$.

A typical experimental run began by equilibrating the surfaces at a separation of several hundred nanometers for about $1 \mathrm{~min}$. Then, we pressed them together with a continuously increasing load. While the surfaces approached each other the liquid was expelled from the gap, first continuously and then in a stepwise fashion. Figure 1 shows the transmitted intensity close to mechanical contact

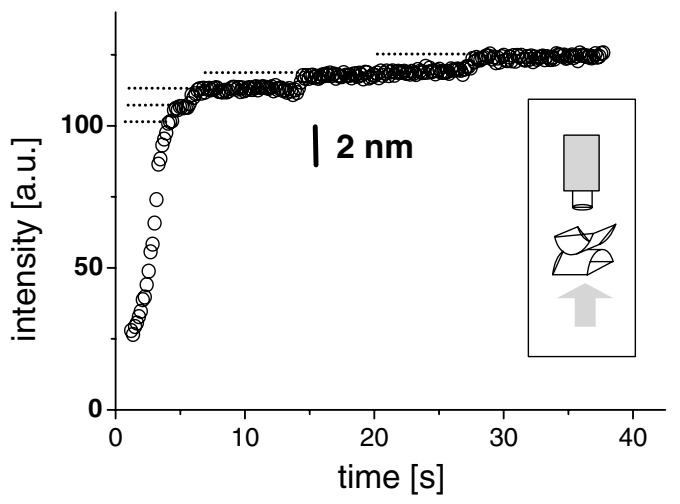

FIG. 1. Transmitted intensity during final stage of lubricant expulsion. The loading rate was $\approx 20 \mathrm{mN} / \mathrm{min}$. The maximum load was $15 \mathrm{mN}$. Inset: Schematic of the experimental setup. 
averaged over an area of $10 \times 10 \mu \mathrm{m}^{2}$ in the center of the contact area. Four discrete steps can be clearly distinguished. Between the steps, the intensity is approximately constant. Using conventional numerical procedures for multilayer thin film interferometry $[10,13]$, we converted the intensity to the thickness of the liquid layer. The reduction of film thickness in each step was found to be $(0.95 \pm 0.1) \mathrm{nm}$. This shows that each step corresponded to the expulsion of one layer of OMCTS molecules, as expected based on earlier experiments [4-7]. The absolute measurement of film thickness was less accurate. Nevertheless, we can give upper and lower limits: For two mica substrates in direct contact one typically observes strong adhesion. This was not the case in the present experiments. Therefore there was at least one layer of OMCTS present at the highest load. As for the upper limit, the extensive literature on oscillatory forcedistance curves with OMCTS [4-7] indicates that films with a thickness of $n>2$ are not stable in the presence of extensive surface flattening at pressures of $\approx 2 \mathrm{MPa}$, as used in the present experiments. We are thus confident that the minimum film thickness at the highest pressure was either two layers or just one. In the following, we will assume the latter.

In contrast to earlier SFA drainage experiments with OMCTS [5], we were able to record two-dimensional images of the dynamics during the expulsion process. Figure 2 shows a series of images recorded during the layering transition around $t=15 \mathrm{~s}$ in Fig. 1. It is clearly seen that a bright area of reduced film thickness $(n-1)$ first appears close to the center of the contact zone and then continuously spreads. Upon nucleation of the $(n-1)$ island, the compressed mica substrates relax inward locally. The elastic relaxation of the wall material converts the applied normal force into a force parallel to the substrates that drives the liquid expulsion. The width of

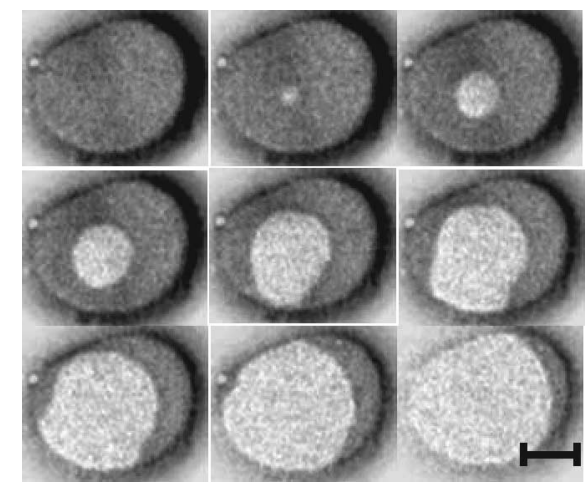

FIG. 2. Dynamics of layer expulsion. Series of images taken around $t \approx 15 \mathrm{~s}$ in Fig. $1 . \Delta t$ between images: $0.3 \mathrm{~s}$. Scale bar: $25 \mu \mathrm{m}$. The ellipsoidal contact zone is gray in the initial state. The bright island appearing in the center is thinner by one monolayer $(\approx 0.95 \mathrm{~nm})$. (Video clips can be viewed at www.wetting.de/sfa.html.) the boundary line between the $n$ and the $(n-1)$ areas was less than our optical resolution $(\approx 1 \mu \mathrm{m})$. From continuum elasticity theory, we expect that it is of the order of the mica thickness, i.e., $400 \mathrm{~nm}$ in the present experiments. Since the gap width assumes noninteger values within this boundary zone, we expect that the layered structure does not prevail there [14]. Figure 3 illustrates this situation schematically for the layer expulsion process $n=3 \rightarrow n=2$.

From the data in Fig. 2 and from the corresponding images of other transitions, we extracted the average radius $r(t)=(A(t) / \pi)^{1 / 2}$ of the area $A(t)$ of thickness $(n-1)$. Figure 4(a) shows the result for a series of four consecutive layering transitions. As a function of film thickness, the average speed of the boundary line assumed values of $37(5 \rightarrow 4), 32(4 \rightarrow 3), 15(3 \rightarrow 2)$, and $3 \mu \mathrm{m} / \mathrm{s}(2 \rightarrow 1)$. This decrease indicates an increase in friction. The corresponding average shear rates decreased from 7800 to $1600 \mathrm{~s}^{-1}$.

In order to extract quantitative information about the viscosity of the liquid, we compared our results to a theoretical model by Persson and Tosatti (PT) $[1,15]$. In this model, the liquid film is treated as a two-dimensional continuum. Its dynamics are governed by the balance between the elastic driving force and a dissipative drag force due to the sliding of the film with respect to the substrate.

$$
\nabla p_{2 \mathrm{D}}=-\rho_{2 \mathrm{D}} \mu_{\mathrm{eff}} \nu .
$$

Here, $p_{2 \mathrm{D}} \propto P d_{0}$ is the two-dimensional pressure and $\nu$ is the flow velocity. $P$ is the applied normal force divided by the contact area $A_{0} . \rho_{2 \mathrm{D}}$ is the two-dimensional mass density and $d_{0}$ the thickness of one monolayer. $\mu_{\text {eff }}$ is the effective drag coefficient. Its value, as determined from a layering transition $n \rightarrow n-1$, characterizes the dissipation within a film of thickness $n$. It was shown earlier that various aspects of the dynamics of layering transitions are correctly described by this model $[9,16]$. Under the assumptions of circular symmetry, homogeneous pressure $P$ across the contact area, and position-independent friction $\mu_{\text {eff }}, A(t)$ is given by the implicit equation [15]

$$
\frac{A(t)}{A_{0}}\left[\ln \left(\frac{A(t)}{A_{0}}\right)-1\right]=-\frac{t}{\tau} .
$$

Here, $\tau=\left(\rho_{2 \mathrm{D}} \mu_{\mathrm{eff}} A_{0}\right) /\left(4 \pi P d_{0}\right)$ is the total time of the transition. The only adjustable parameter in this formula is $\mu_{\text {eff }}$. The fit curves are plotted as solid lines in Fig. 4(a). The agreement with the experimental data shows that

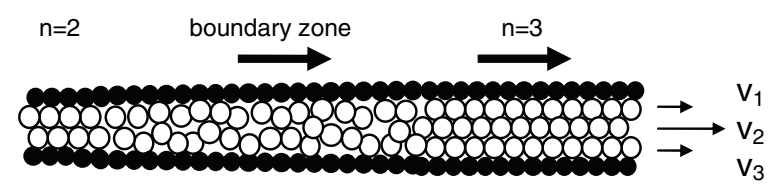

FIG. 3. Schematic of an expulsion process $n=3 \rightarrow n=2$. 
a)

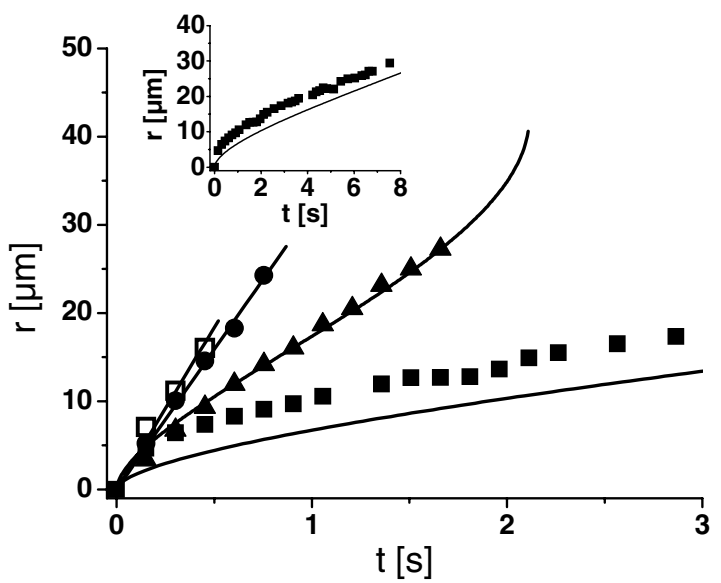

b)

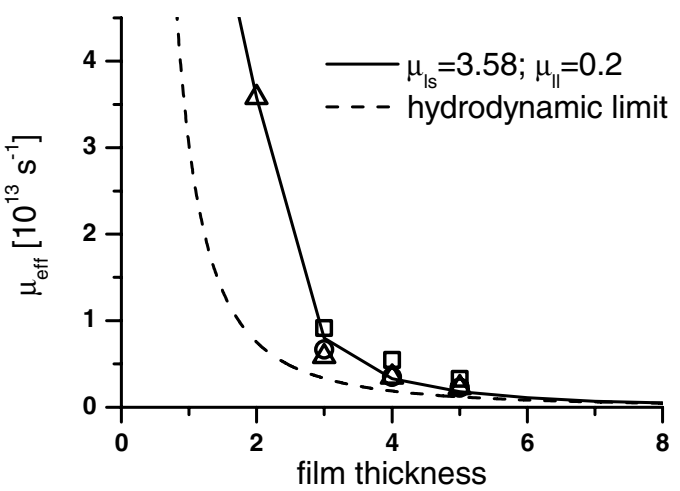

FIG. 4. Evaluation of the effective drag coefficient. (a) Effective radius $r(t)$ of the $(n-1)$ layer island for a series of consecutive layering transitions. Symbols: experimental data; lines: theoretical fits using Eq. (1). Main figure: transitions $5 \rightarrow 4$ (open squares), $4 \rightarrow 3$ (circles), $3 \rightarrow 2$ (up triangles; taken from the sequence in Fig. 2), $2 \rightarrow 1$ (filled squares). Inset: $2 \rightarrow 1$ transition on a different time scale. We attribute the deviation between theory and experiment for this transition to deviations from circular symmetry. (b) $\mu_{\text {eff }}$ vs film thickness (in units of $d_{0}$ ). Different symbols refer to data recorded at different locations on the same set of mica surfaces. The solid line shows the result of our model with best fit values of $\mu_{l s}$ and $\mu_{l l}$ (in units of $10^{13} \mathrm{~s}^{-1}$ ). The dashed line represents the asymptotic continuum limit.

squeeze out dynamics can be described by the laws of two-dimensional hydrodynamics [17]. Figure 4(b) shows best fit values for $\mu_{\text {eff }}$ as a function of $n$.

In order to understand the increase of $\mu_{\text {eff }}$ with decreasing $n$, we consider the dynamics within the film on a microscopic level. During the layering transitions, the boundary zone propagates across the contact area (from left to right in Fig. 3). There are three processes involving these dynamics: (i) At the leading edge of the boundary zone, the well-defined $n$-layer structure disappears and the film becomes disordered. (ii) At the trailing edge, the disordered structure of the boundary zone trans- forms back into a well-defined layer structure with thickness $n-1$. (iii) Because of mass conservation, the material in the area of thickness $n$ ahead of the boundary line flows towards the edge of the contact area. [In contrast, the material in the $(n-1)$ area is at rest.] In principle, all these processes give rise to dissipation. However, the former two are related only to the boundary line, which is a one-dimensional object. The last process involves transport everywhere in the (much larger) twodimensional area of thickness $n$. Therefore, we assume that process (iii) dominates the dissipation. Implicitly, this assumption was also used in the PT model [15].

The thickness dependence of $\mu_{\text {eff }}$ can be understood by extending the original PT model to films consisting of $n$ independent liquid layers. We assume that equations analogous to Eq. (1) hold for each layer independently. Coupling between adjacent layers is provided by analogous viscous drag terms [18]. Furthermore, we assume that the two-dimensional pressure $p_{2 \mathrm{D}}$ is distributed evenly between the molecular layers, i.e., the stress acting on each layer is given by $p_{2 \mathrm{D}}^{\prime}=p_{2 \mathrm{D}} / n$. The velocity $\nu_{i}$ of the $i$ th layer is then determined by

$$
\nabla p_{2 \mathrm{D}}^{\prime}=\rho_{2 \mathrm{D}}\left[\mu_{i, i-1}\left(\nu_{i}-\nu_{i-1}\right)+\mu_{i, i+1}\left(\nu_{i}-\nu_{i+1}\right)\right],
$$

with $i=1 \cdots n$. Here, $\mu_{i, i \pm 1}$ is the drag coefficient between layer $i$ and $i \pm 1$. We introduce two different drag coefficients $\mu_{l s}$ and $\mu_{l l}$ to describe the friction between the solid substrates and the adjacent liquid layers and the mutual friction between two adjacent liquid layers, respectively; i.e., $\mu_{1,0}=\mu_{n, n+1}=\mu_{l s}$ and $\mu_{i, i-1}=$ $\mu_{i, i+1}=\mu_{l l}$ otherwise. This set of equations can be solved easily for the velocities $\nu_{i}$. The velocity of the boundary line, which is the experimentally observed quantity, is given by $\nu_{b}=\sum_{i} \nu_{i}(i=1 \cdots n)$ due to mass conservation. The $\nu_{i}$ 's follow a parabolic profile. For $n \gg 1$, this discrete profile approaches the analytical hydrodynamic solution for a Poiseuille flow $\nu(z)=$ $\left((d / 2)^{2}-z^{2}\right) /(2 \eta) \nabla p+\nu_{\text {slip }}$. Here, $\eta$ is the bulk viscosity of the liquid, $z$ is the coordinate perpendicular to the surfaces, and $d$ is the film thickness. $\nu_{\text {slip }}=\nu_{1}=\nu_{n}$ is the slip velocity [19]].

From Eq. (1), we find $\mu_{\text {eff }}(n)=\left|\nabla p_{2 \mathrm{D}} /\left(\rho_{2 \mathrm{D}} \sum_{i} \nu_{i}\right)\right|$, which is an implicit function of both $\mu_{l l}$ and $\mu_{l s}$. Best fit values of $\mu_{l s}=(3.58 \pm 0.3) \times 10^{13} \mathrm{~s}^{-1}$ and $\mu_{l l}=$ $(0.2 \pm 0.04) \times 10^{13} \mathrm{~s}^{-1}$ were obtained by minimizing numerically the mean square deviation between the model function and the experimental data. The result is shown as a solid line in Fig. 4(b). Since $\mu_{l l}$ does not depend on $n$ in our model, $\mu_{\text {eff }}(n)$ should converge for $n \rightarrow \infty$ towards the flow resistance $\mu(d)$ of a thin liquid film calculated in the hydrodynamic limit [20]. The latter is given by $\mu(d)=12 \eta /\left(\rho d^{2}\right)$ [dashed line in Fig. 4(b)]. In this limit, one obtains $\mu_{l l \text {-hydro }}=h /\left(\rho_{2 \mathrm{D}} d_{0}\right)=0.3 \times$ $10^{13} \mathrm{~s}^{-1}$, which is remarkably close to the best fit value obtained above. This means that the momentum transfer 
between adjacent liquid layers in thin films with welldefined positional ordering perpendicular to the solid surfaces is of the same order of magnitude as the coupling between equivalent "layers" of the same thickness in a bulk liquid with random positions in all three dimensions. These findings are in qualitative agreement with recent nonequilibrium molecular dynamics simulations [21]. Apart from the $d^{-2}$ dependence, which is due to the two-dimensional character of the model, the increase of $\mu_{\text {eff }}$ with decreasing film thickness thus arises only from the increasing weight of $\mu_{l s}$ compared to $\mu_{l l}$ [22].

There is an ongoing debate in the literature, whether thin liquid films solidify [6] under confinement and/or to what extent their dynamics are slowed down [7,23]. Obviously, we explained our experimental observations for the same system without alluding to confinementinduced solidification. However, the present experiments refer to a dynamic situation. It is conceivable that the films are solidified in equilibrium, but liquefied under the conditions of the present experiments. This requires that the critical shear stress $\sigma_{c}$ required to either shear melt such solidified layers or to depin them from the solid substrates is smaller than $\nabla p_{2 \mathrm{D}}$ in our experiments, i.e., $\sigma_{c}<\nabla p_{2 \mathrm{D}} \approx P d_{0} / R \approx 0.1 \cdots 1 \mathrm{kPa}$ [24]. If solidified in equilibrium, thin OMCTS layers would thus be extremely soft solids.

We thank S. Herminghaus, B. Persson, S. Granick, and J. Klein for fruitful discussions and comments. This work was funded by the German Science Foundation within the priority program "Wetting and Structure Formation at Interfaces" (Grant No. Mu 1472/2).

*Corresponding author.

Email address: frieder.mugele@physik.uni-ulm.de

[1] B. N. J. Persson, in Sliding Friction, edited by R. Wiesendanger and $K$. von Klitzing, NanoScience and Technology (Springer-Verlag, Berlin, 2000), 2nd ed.

[2] See, for example, V.S. J. Craig, C. Neto, and D. R. M. Williams, Phys. Rev. Lett. 87, 054504 (2001); Y. Zhu and S. Granick, Phys. Rev. Lett. 87, 096104 (2001).

[3] J. N. Israelachvili, Intermolecular and Surface Forces (Academic Press, London, 1992), 2nd ed.

[4] R. G. Horn and J. N. Israelachvili, J. Chem. Phys. 75, 1400 (1981); H. K. Christenson and C. E. Blom, J. Chem. Phys. 86, 419 (1987).

[5] D. Y. C. Chan and R. G. Horn, J. Chem. Phys. 83, 5311 (1985).

[6] J. Klein and E. Kumacheva, Science 269, 816 (1995); J. Chem. Phys. 108, 6996 (1998); E. Kumacheva and J. Klein, J. Chem. Phys. 108, 7010 (1998).

[7] A. L. Demirel and S. Granick, Phys. Rev. Lett. 77, 2261 (1996).

[8] J. N. Israelachvili, P. M. McGuiggan, and A. M. Homola, Science 240, 189 (1988).

[9] F. Mugele and M. Salmeron, Phys. Rev. Lett. 84, 5796 (2000).
[10] F. Mugele, T. Becker, A. Klingner, and M. Salmeron, Colloids Surf. A 206, 105 (2002).

[11] The creation of nanoparticles in the conventional mica surface preparation procedure and their impact on surface forces measurements were described in a series of recent publications: S. Ohnishi, M. Hato, K. Tamada, and H. K. Christenson, Langmuir 15, 3312 (1999); M. M. Kohonen, F. C. Meldrum, and H. K. Christenson, Langmuir 19, 975 (2003); M. Heuberger and M. Zäch, Langmuir 19, 1943 (2003); Z. Lin and S. Granick, Langmuir 19, 7061 (2003).

[12] P. Frantz and M. Salmeron, Tribol. Lett. 5, 151 (1998).

[13] M. Born and E. Wolf, Principles of Optics (Cambridge University Press, Cambridge, 1999), 6th ed.

[14] See, for example, J. Gao, W. D. Luedtke, and U. Landman, Phys. Rev. Lett. 79, 705 (1997); M. Schoen and D. J. Diestler, Phys. Rev. E 56, 4427 (1997).

[15] B. N. J. Persson and E. Tosatti, Phys. Rev. B 50, 5590 (1994).

[16] S. Zilberman, B. N. J. Persson, A. Nitzan, F. Mugele, and M. Salmeron, Phys. Rev. E 63, 055103 (2001).

[17] Recent theoretical work shows that the agreement still holds if the (correct) Hertzian pressure distribution is taken into account [S. Zilberman, T. Becker, F. Mugele, B. N. J. Persson, and A. Nitzan, J. Chem. Phys. 118, 11160 (2003)]. The correction merely shifts the value of $\tau$ (and thus $\mu_{\text {eff }}$ ) by about $20 \%$.

[18] P. G. de Gennes and A. M. Cazabat, C.R. Acad. Sci. 310, 1601 (1990).

[19] From Eq. (3), it follows immediately that $\nu_{\text {slip }}=$ $\left(\nabla p_{2 \mathrm{D}}^{\prime} /\left(2 \rho_{2 \mathrm{D}} \mu_{l s}\right)\right.$, i.e., $\nu_{\text {slip }}$ vanishes for $\mu_{l s} \rightarrow \infty$, as expected. We found that the slip length $\delta=\nu_{\text {slip }} / d \nu / d z$ is smaller than the molecular diameter under the conditions of the present experiments [20].

[20] Detailed calculations will be described elsewhere [F. Mugele (to be published)].

[21] P. A. Thompson and M. O. Robbins, Phys. Rev. A 41, 6830 (1990); L. Pozhar, Phys. Rev. E 61, 1432 (2000); H. Zhang, B. J. Zhang, S. Liang, Y. Lu, W. Hu, and Z. Jin, Chem. Phys. Lett. 350, 247 ( 2001).

[22] These conclusions are independent of whether the minimum value of $n$ in the present experiments is one or two monolayers. If we repeat the analysis assuming the latter, we find $\mu_{l l}=(0.53 \pm 0.05) \times 10^{13} \mathrm{~s}^{-1}$ and $\mu_{l s} \geq$ $3 \times 10^{15} \mathrm{~s}^{-1}$, i.e., $\mu_{l l}$ is still of the same order of magnitude as $\mu_{l l \text {,hydro }}$, while $\mu_{l s}$ is so large that the first layer is essentially immobile.

[23] A. Mukhopadhyay, J. Zhao, S. C. Bae, and S. Granick, Phys. Rev. Lett. 89, 136103 (2002).

[24] According to numerical simulations [B. N. J. Persson, V. N. Samoilov, S. Zilberman, and A. Nitzan, J. Chem. Phys. 117, 3897 (2002)], the presence of pinning sites on the surfaces increases the static friction coefficient of confined liquids significantly. The extremely small value for $\sigma_{c}$ in our experiments may thus be related to our surface preparation procedure, which eliminates potential artifacts due to nanoparticles, such as described in [11,12]. In addition, the fact that we did not distill the liquid (in contrast to [6]) may also have played a role. 